Check for updates

Cite this: RSC Adv., 2019, 9, 35939

Received 22nd August 2019

Accepted 10th October 2019

DOI: $10.1039 / c 9 r a 06629 b$

rsc.li/rsc-advances

\section{Defect-related luminescent nanostructured hydroxyapatite promotes mineralization through both intracellular and extracellular pathways}

\begin{abstract}
Chunyan Dai, ${ }^{\text {ab }}$ Linhua Zhu, (D)*ab Guangying Chen*a and David M. Haddleton (D) *b
Hydroxyapatite (HAP) is a widely used biomaterial for bone tissue substitution due to its chemical similarity with the natural bone. Defect-related luminescent HAP materials have the same chemical composition as normal HAP and excellent biocompatibility. However, only few works have focused on the defect-related luminescent HAP materials on bone regeneration. In this work, we systematically investigated the bone regeneration pathway induced by nanostructured particles using defect-related luminescent hydroxyapatite (S2) materials. We monitored the subcellular distribution and location of S2 during osteoblast differentiation with the property of defect-related luminescence. Nano-scale S2 could be internalized by osteoblasts (OBs) via caveolae-mediated endocytosis and macropinocytosis. S2 incorporated into the lysosomes dissolved and released calcium ions for the formation of mineralized nodules. Extracellular S2 also promoted bone regeneration as a nucleation site. Taken together, the physical properties of hydroxyapatite control the bone regeneration pathway in osteoblasts.
\end{abstract}

\section{Introduction}

Hydroxyapatite (HAP) is the major mineral component of calcified tissues. Synthetic HAP $\left(\mathrm{Ca}_{10}\left(\mathrm{PO}_{4}\right)_{6}(\mathrm{OH})_{2}\right)$ has been extensively used as an implant material for bone substitutes due to its biocompatibility, bioactivity and osteoconduction properties. ${ }^{1-3}$ To monitor the biological relevant testing of HAP, it is desirable to develop fluorescent labeled nanoparticles for tracing. However, traditional HAP generally exhibits no luminescence, which seriously limits its applications. To overcome this limitation, fluorescent HAP nanoparticles doped with rare earth ions (e.g., $\mathrm{Eu}^{3+}$ and $\mathrm{Tb}^{3+}$ ) and organic dyes have been developed and applied in the bioimaging field. ${ }^{4-6}$ However, the introduction of these doped ions and organic dyes may affect the mineralization of bone cells, which is unsuitable for studying the effects of HAP on bone regeneration. Thus, it is necessary to develop novel luminescent HAP without introducing rare earth ions and fluorescent dyes.

Defect-related luminescent materials do not contain any transition metal or rare earth activators, and the emission arises from special defects. ${ }^{7}$ These materials were earlier reported in $1986^{8}$ and have achieved remarkable development since the synthesis of white phosphors from a silicate-carboxylate sol-gel precursor. ${ }^{9}$ The defect-related luminescent materials can be

${ }^{a}$ Key Laboratory of Tropical Medicinal Plant Chemistry of Ministry of Education, Hainan Normal University, Haikou 571158, P.R. China. E-mail: zhulinhua1981@ 163.com; chyying123@163.com

${ }^{b}$ Department of Chemistry, University of Warwick, Coventry, CV4 7AL, UK. E-mail: d.m. haddleton@warwick.ac.uk used in various electronics-related applications due to the advantages of low toxicity and stability. ${ }^{10,11}$ In our previous work, three defect-related luminescent hydroxyapatite particles with different morphologies and sizes were synthesized. The defect-related luminescent HAP materials in the nano-scale show excellent biocompatibility in biomedical applications. ${ }^{12}$ The defect-related luminescent materials are nontoxic for osteoblasts. Previous studies have reported that the materials modified with HAP tend to improve the mineralization of bone cells. ${ }^{13}$ Thus, we want to know whether the defect-related luminescent HAP materials have any effects on bone regeneration.

In this current work, by taking advantage of the defectrelated luminescence properties, an evaluation of the HAP nanoparticles on the subcellular location and their influence on the bone regeneration of osteoblasts was carried out systematically. This work is quite different from our previous work, and it is an extension of the last work.

\section{Materials and methods}

\section{Materials}

Newborn (1-2 days old) ICR mice were purchased from the Experimental Animal Center of Hebei Medical University. DMEM was purchased from Gibco-BRL (Grand Island, NY, USA). Neonatal bovine serum (NBS) was obtained from Hangzhou Sijiqing Biological Engineering Materials Co., Ltd. (Hangzhou, China). Trypsin, benzylpenicillin, streptomycin, $\beta$ glycerphosphate, ascorbic acid, dexamethasone, sirius red, picric acid, alizarin red S (ARS), cetylpyridinium-chloride, 
propidium iodide (PI) and acridine orange (AO) were purchased from Sigma-Aldrich (St. Louis, MO, USA). Mito-Tracker Green, Lyso-Tracker Red, and wortmannin were purchased from Beyotime Institute of Biotechnology (Haimen, China). Nystatin was purchased from Aladdin (Shanghai, China). Chlorpromazine hydrochloride was purchased from TCI (Tokyo, Japan). An ALP activity kit and a micro-protein assay kit were obtained from Nanjing Jiancheng Biological Engineering Institute (Nanjing, China). TRIzol reagent was purchased from Invitrogen (Carlsbad, CA, USA) The Revertaid First Strand cDNA Synthesis Kit was purchased from Thermo Scientific (Vilnius, Lithuania). UltraSYBR mixture was purchased from Beijing ComWin Biotech Co., Ltd. (Beijing, China). All the other chemicals were of analytical grade and used without further purification.

\section{Synthesis of HAP}

A defect-related luminescent HAP sample was synthesized as previously reported. ${ }^{12} \mathrm{Ca}\left(\mathrm{NO}_{3}\right)_{2} \cdot 4 \mathrm{H}_{2} \mathrm{O}$ and hexadecyltrimethylammonium bromide (CTAB) were dissolved in deionized water, and the $\mathrm{pH}$ was adjusted to 7.0 using $\mathrm{HNO}_{3}$ or $\mathrm{NH}_{3} \cdot \mathrm{H}_{2} \mathrm{O}$ solutions. Subsequently, a "mixing solution" containing trisodium citrate and $\left(\mathrm{NH}_{4}\right)_{2} \mathrm{HPO}_{4}$ was added to the above solution. After stirring for $20 \mathrm{~min}$, the mixture was transferred into an autoclave, sealed, and maintained at $180^{\circ} \mathrm{C}$ for $24 \mathrm{~h}$. Precipitates were washed with deionized water and absolute ethanol and then air-dried at room temperature. The HAP sample prepared at $\mathrm{pH} 7.0$ was labeled as $\mathrm{S} 2$.

\section{Characterization}

The morphology of S2 was determined using a JSM-7500 F cold field scanning electron microscope (SEM) (JEOL, Japan) and an FEI Tecnai G2 S-Twin transmission electron microscope (TEM) (FEI, America). The chemical structure was characterized by a Bruker Advance D8 X-Ray diffractometer (XRD) (Bruker, Germany). Photoluminescence (PL) spectra of S2 were tested by a Hitachi F-7000 spectrophotometer.

\section{Isolation and culture of primary OBs}

Primary OBs were isolated from neonatal mouse calvaria by sequential digestion with trypsin and collagenase as described previously. ${ }^{12}$ All studies involving the use of animals were conducted in conformity with NIH guidelines (NIH Publications No. 8023, revised 1978) and were approved by Animal Care and Use Committee of Hebei Medical University. Briefly, calvaria were isolated aseptically from neonatal ICR mice. After eliminating all soft tissues, the calvaria were digested once with $0.25 \%$ trypsin for $25 \mathrm{~min}$ and twice with $0.1 \%$ collagenase II for $1 \mathrm{~h}$ each. The cells were collected and cultured in a $75 \mathrm{~cm}^{2}$ flask with complete medium (DMEM containing 10\% NBS, $100 \mathrm{U}$ $\mathrm{mL}^{-1}$ penicillin, and $100 \mu \mathrm{g} \mathrm{mL} \mathrm{g}^{-1}$ streptomycin) at $37^{\circ} \mathrm{C}$ under a humidified atmosphere with $5 \% \mathrm{CO}_{2}$. The medium was changed every 3 days.

\section{In Vitro cell uptake}

The uptake of S2 was performed according to the method described by Amornwachirabodee et al. ${ }^{14}$ In brief, OBs were seeded in a $35 \mathrm{~mm}$ glass bottom dish at the density of $4 \times 10^{4}$ cells per well and allowed to adhere overnight. Cells were incubated with S2 at $37^{\circ} \mathrm{C}$ under a humidified atmosphere with $5 \% \mathrm{CO}_{2}$ for $24 \mathrm{~h}$. The media were removed, and the cells were washed thrice with PBS; then, the cells were stained with $6 \mu \mathrm{g}$ $\mathrm{mL}^{-1}$ acridine orange (AO) for $30 \mathrm{~min}$ in the dark. Then, the media were removed, and the cells were washed thrice with PBS before being subjected to confocal laser scanning microscopy (CLSM) (Olympus FV1000, Japan). Excitation at $488 \mathrm{~nm}$ and fluorescence signals at $580-680 \mathrm{~nm}$ were collected as AO fluorescence signals. Excitation at $405 \mathrm{~nm}$ and fluorescence signals at $415-515 \mathrm{~nm}$ were collected as S2 fluorescence signals. Images indicating the locations of S2 were then constructed using the overlap signals.

\section{Inhibition of endocytosis}

The endocytotic pathways were performed according to the method described by Greulich et al. ${ }^{15}$ In brief, OBs were seeded in 6-well plates at the density of $1 \times 10^{5}$ cells per well and allowed to adhere overnight. OBs were treated with inhibitors of endocytosis $(3.5 \mu \mathrm{M}$ chlorpromazine hydrochloride, $5 \mu \mathrm{M}$ nystatin or $200 \mathrm{nM}$ wortmannin) for $30 \mathrm{~min}$ at $37^{\circ} \mathrm{C}$ under cell culture conditions. Then, OBs were washed twice with PBS and changed for a new complete medium. After this pretreatment, S2 was added to the culture (final concentration: $80 \mu \mathrm{g} \mathrm{mL} \mathrm{m}^{-1}$ ) and the cells were incubated for a further $1 \mathrm{~h}$. Cells treated without inhibitors were set as the control. OBs incubated with S2 were set as a positive control, and the cells incubated without S2 were set as a negative control. Subsequently, the cells were harvested and resuspended in PBS. The uptake of particles into OBs was analyzed by a flow cytometer (FACSCalibur, Becton Dickinson). The side scatter data were analyzed using the CellQuest software. Calibration reagents and solutions for flow cytometry were obtained from Becton Dickinson. After achieving the appropriate instrument settings and compensations, the instrument setup was not changed throughout the study. Ten thousand cells were acquired for each measurement.

\section{Subcellular location of S2}

The co-localization studies were performed according to the method described by Greulich et al. ${ }^{15}$ OBs were cultured as described above and exposed to $80 \mu \mathrm{g} \mathrm{ml} \mathrm{m}^{-1} \mathrm{~S} 2$ particles for $24 \mathrm{~h}$. Following incubation, the OBs were labeled with specific cell organelle fluorescent probes (Beyotime). In order to label the lysosomes and mitochondrion, the cells were incubated with $75 \mathrm{nM}$ Lyso-Tracker Red and $100 \mathrm{nM}$ Mito-Tracker Green in complete medium for $45 \mathrm{~min}$ at $37{ }^{\circ} \mathrm{C}$. CLSM was taken (FV1000, Olympus) and processed using the FV10-ASW software. To label the cell nucleus, the cells were fixed with $4 \%$ paraformaldehyde for $10 \mathrm{~min}$, followed by permeabilization with $0.2 \%$ Triton X-100 in PBS for 2 min. Then, the cells were incubated with $100 \mu \mathrm{g} \mathrm{mL}{ }^{-1} \mathrm{PI}$ in complete medium for $30 \mathrm{~min}$ 
at $37^{\circ} \mathrm{C}$. After three rinses in PBS, the cells were viewed under CLSM (Olympus).

\section{Collagen production analysis}

The Sirius red staining-based colorimetric assay was used to quantify collagen deposition. Collagen production analysis was performed according to the method described by Walter et al. ${ }^{16}$ OBs were seeded in 48-well tissue culture plates at the density of $1 \times 10^{4}$ cells per well with OS. S2 at a final concentration of 80 $\mu \mathrm{g} \mathrm{mL} \mathrm{m}^{-1}$ was added to complete medium and incubated for 14 d. OBs treated with only OS were used as the control group. The plates were washed three times with ice-cold PBS and fixed for $1 \mathrm{~h}$ with $10 \%$ formaldehyde and washed with triple-distilled water. Then, $200 \mu \mathrm{L}$ of dye solution $(1 \mathrm{mg}$ Sirius red was dissolved in $1 \mathrm{~mL}$ saturated solution of picric acid) per well was added and incubated for $1 \mathrm{~h}$. After that, plates were washed with $0.01 \mathrm{M} \mathrm{HCl}$ to remove the excess of the dye solution. The collagen fixed with dye was extracted with $200 \mu \mathrm{L}$ of $0.1 \mathrm{M} \mathrm{NaOH}$ solution per well. OD was measured by a microplate spectrophotometer at $550 \mathrm{~nm}$. All results were normalized by the protein content. The collagen production rate (\%) was calculated using the following formula: $\left(\mathrm{OD}_{\text {treated }}-\mathrm{OD}_{\text {blank }}\right) /$ $\left(\mathrm{OD}_{\text {control }}-\mathrm{OD}_{\text {blank }}\right) \times 100$.

\section{Mineralized matrix formation assay}

An ARS staining assay was used to evaluate mineralized matrix formation, as described in detail elsewhere. ${ }^{17}$ OBs were seeded in 48-well culture plates at the density of $1 \times 10^{4}$ cells per well with the osteogenic induction supplement (OS) containing 0.1 $\mu \mathrm{M}$ dexamethasone, $5.0 \mathrm{mM} \beta$-glycerophosphate and $50 \mu \mathrm{g}$ $\mathrm{mL}^{-1}$ ascorbic acid. The medium was changed to differentiation medium OS in the presence or absence of S2 at the concentrations of 20,40 and $80 \mu \mathrm{g} \mathrm{mL} \mathrm{m}^{-1}$ for $17 \mathrm{~d}$. OBs treated with only OS were used as the control group. After incubation, the cells were fixed in $95 \%$ ethanol for $10 \mathrm{~min}$ at room temperature. The fixed cells were washed with PBS and stained with ARS ( $40 \mathrm{mM}$ ) for $30 \mathrm{~min}$ at $37^{\circ} \mathrm{C}$. Quantitation of ARS staining was performed by elution with $10 \%(\mathrm{w} / \mathrm{v})$ cetylpyridinium chloride $200 \mu \mathrm{L}$ per well at room temperature for $20 \mathrm{~min}$. OD was measured by a microplate spectrophotometer at $570 \mathrm{~nm}$. All results were normalized by the protein content. The mineralized rate (\%) was calculated using the following formula: $\left(\mathrm{OD}_{\text {treated }}-\mathrm{OD}_{\text {blank }}\right) /\left(\mathrm{OD}_{\text {control }}-\mathrm{OD}_{\text {blank }}\right) \times 100$.

\section{Real-time RT-PCR}

The quantitative messenger RNA (mRNA) expression of runtrelated transcription factor 2 (RUNX2) and osteocalcin (OCN) was characterized by real-time reverse transcriptase polymerase chain reaction (RT-PCR) (ABI Stepone Plus, USA). After 14 days of culture, total RNA was extracted using the TRIzol reagent according to the manufacturer's instructions. The quality and concentration of RNA samples were detected using a spectrophotometer (Bio-Rad Smartspec Plus, USA) at 260, $280 \mathrm{~nm}$ absorbance and calibrated by DEPC water. cDNA was reverse transcribed using the Revertaid First Strand cDNA Synthesis Kit. The resultant cDNA was used for the PCR reaction using the
UltraSYBR mixture. Reaction conditions were $95{ }^{\circ} \mathrm{C}$ for $15 \mathrm{~s}$, $60{ }^{\circ} \mathrm{C}$ for $30 \mathrm{~s}$ and $72{ }^{\circ} \mathrm{C}$ for $30 \mathrm{~s}$. Three independent cultures were analyzed. Gene expression was normalized by glyceraldehyde 3-phosphate dehydrogenase (GAPDH) using a comparative cycle threshold $\left(\Delta \Delta C_{\mathrm{t}}\right)$ method. Primer pairs used were as follows: GAPDH forward: $5^{\prime}$-GAC TTC AAC AGC AAC TCC CAC$3^{\prime}$, reverse: $5^{\prime}$-TCC ACC ACC CTG TTG CTG TA-3'; OCN forward: 5'-GAA CAG ACT CCG GCG CTA-3', reverse: 5'-AGG GAG GAT CAA GTC CCG-3'; RUNX2 forward: $5^{\prime}$-TTC TCC AAC CCA CGA ATG CAC-3', reverse: 5'-CAG GTA CGT GTG GTA GTG AGT-3'.

\section{Nucleation role of $\mathbf{S 2}$ in the formation of the mineralized matrix}

ARS staining assay was used to stain the mineralized matrix, as described above. In brief, cells were seeded in a glass coverslip at $2 \times 10^{4}$ cells per $\mathrm{mL}$ and cultured for $24 \mathrm{~h}$. After treatment with OS in the presence or absence of S2 $\left(80 \mu \mathrm{g} \mathrm{mL} L^{-1}\right)$ for $17 \mathrm{~d}$, cells were stained with ARS as described above. The cells were washed three times with PBS. The mineralized matrix was detected by a fluorescence microscope (Olympus BX53, Japan) with an excitation of 395-410 $\mathrm{nm}$ and an emission of 450$470 \mathrm{~nm}$ for S2 to trace the fate of HAP.

\section{Statistical analysis}

Data were collected from three separate experiments. The results were expressed as mean \pm standard deviation (SD). The statistical differences were analyzed using SPSS $^{\prime} t$-test. $P<0.05$ was considered to indicate statistical differences.

\section{Results and discussion}

\section{Characterization of $\mathrm{S} 2$}

In our experiments, defect-related luminescent hydroxyapatite particles S2 were synthesized according to our previously reported method, in which the fabrication process has been described in detail. Sample S2 particles were formed as nanorods (Fig. 1a and b). The diameter and length of $\mathrm{S} 2$ were approximately $25 \mathrm{~nm}$ and $100 \mathrm{~nm}$, respectively. Fig. 1c shows that the diffraction peaks of S2 can be well indexed to the hexagonal phase HAP (JCPDS card no. 09-0432). The PL excitation and emission spectra (Fig. 1d) of the S2 nanorods display broad bands centered at 339 and $418 \mathrm{~nm}$, respectively.

\section{Uptake and mechanism of S2 in OBs}

Nanophase HAP possesses unique properties and many researchers have found that the HAP nanoparticles can easily enter many types of cells. ${ }^{18,19}$ Strong blue fluorescence was observed inside the OBs incubated with nano-scale S2 for $24 \mathrm{~h}$ (Fig. 2). Furthermore, most of the fluorescence signals were within the cytoplasm. It could be confirmed that the S2 nanorods entered OBs.

In order to analyze the mechanism of the cellular uptake of nano-scale HAP, selective inhibitors of the specific routes of endocytosis were applied. OBs were preincubated for $30 \mathrm{~min}$ with individual inhibitors; then, $\mathrm{S} 2\left(80 \mu \mathrm{g} \mathrm{mL} \mathrm{m}^{-1}\right)$ was added and 
a

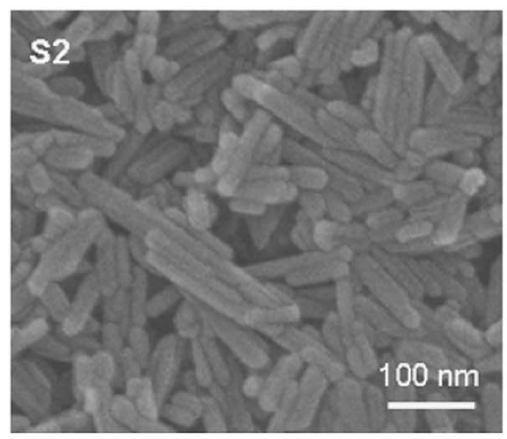

b

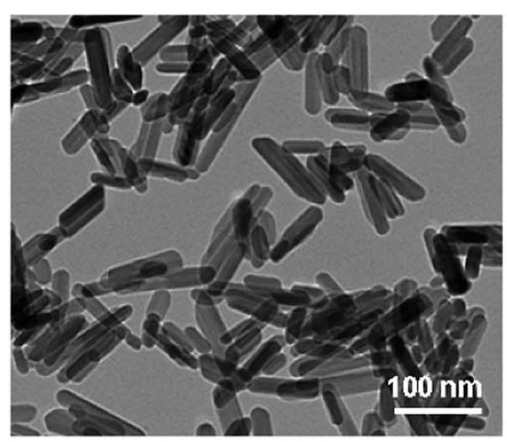

c

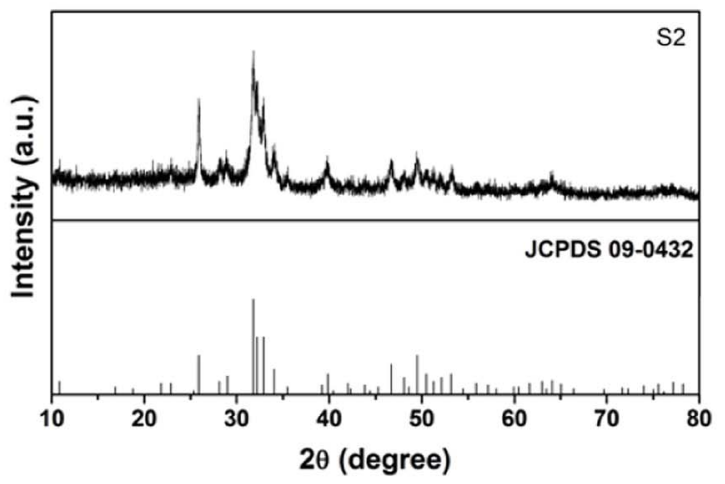

d

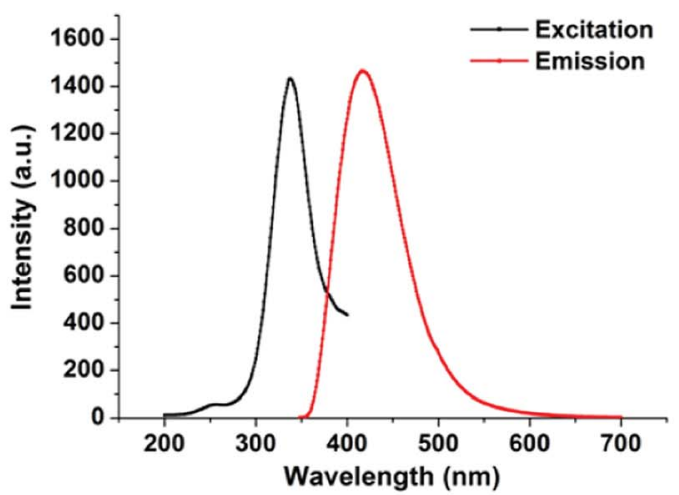

Fig. 1 (a) SEM image of S2. (b) TEM image of S2. (c) XRD pattern of S2. (d) PL excitation and emission spectra of S2.

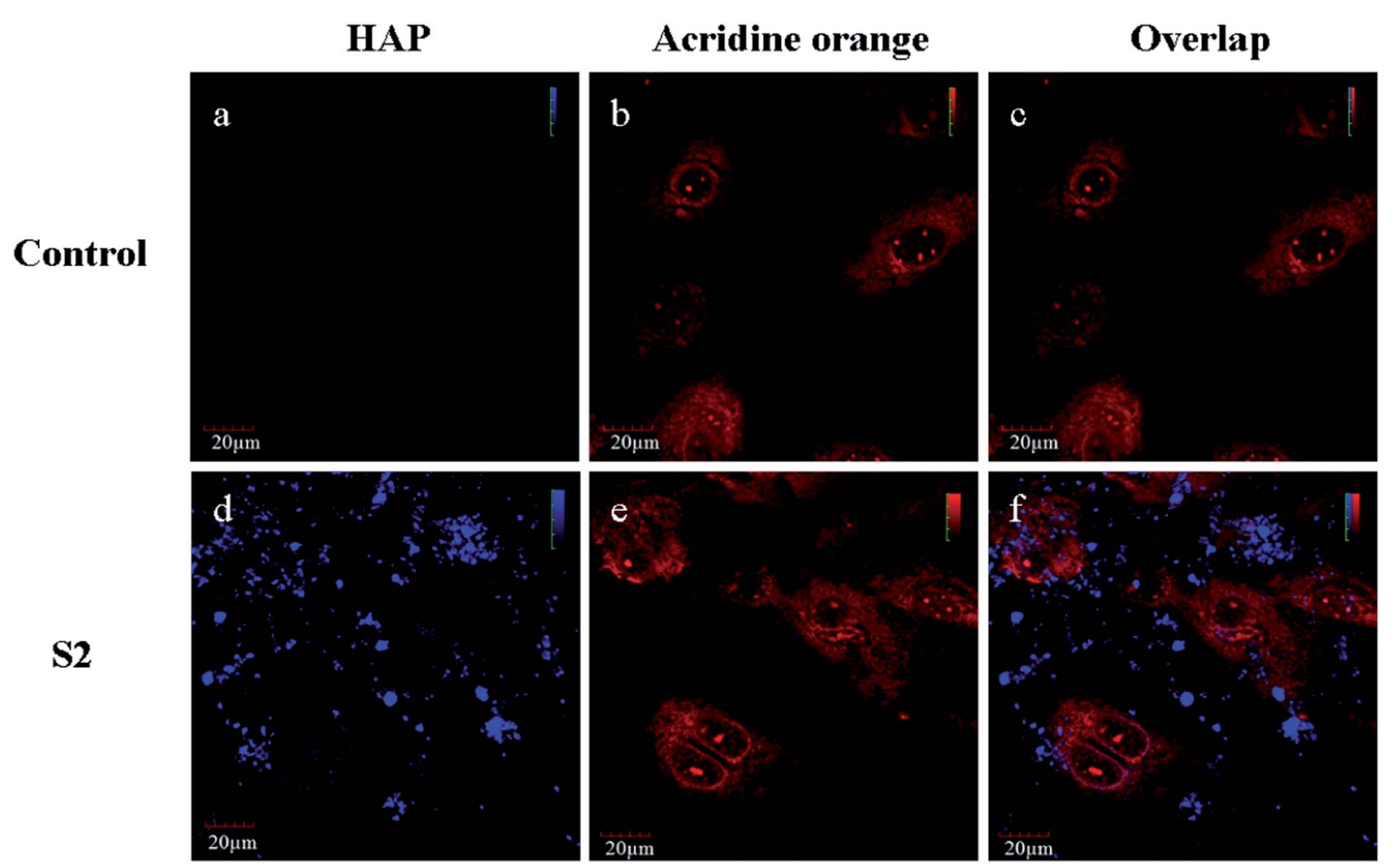

Fig. 2 Evaluation of the uptake of S2 by OBs. (a-c) Control, $(d-f)$ S2. (a and d) Blue fluorescence (425-475 nm) images from S2 by excitation at $405 \mathrm{~nm}$ with a semiconductor laser beam. (b and e) Red fluorescence $(617-717 \mathrm{~nm}$ ) images from AO stained at $488 \mathrm{~nm}$ with an argon laser beam. (c and f) The overlap images are nanorods and cells; scale bar is $20 \mu \mathrm{m}$. 


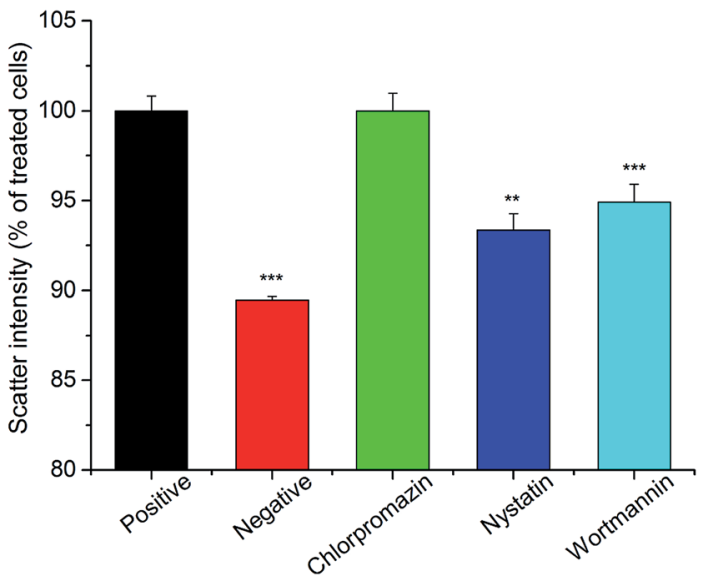

Fig. 3 Uptake of S2 into OBs in the presence of different endocytosis inhibitors. OBs were preincubated for $30 \mathrm{~min}$ with the individual inhibitors and subsequently, the S2 $\left(80 \mu \mathrm{g} \mathrm{mL}^{-1}\right)$ uptake over $1 \mathrm{~h}$ was analyzed by flow cytometric light scatter analysis; the representative scattergrams are shown.

cell culture was continued for $1 \mathrm{~h}$ (Fig. 3). The quantification of the uptake of nanoparticles was performed by flow cytometry using side scatter analysis. Compared to the result for the negative control, the scatter signal significantly increased in the treatment of S2. Furthermore, the addition of inhibitors via clathrin-mediated endocytosis OBs treated with S2 and a specific clathrin-mediated endocytosis inhibitor (chlorpromazine) showed no significant decrease in the scatter signal. In the presence of a specific caveolae inhibitor (nystatin) and macropinocytosis inhibitor (wortmannin), the scatter signal decreased $(6.64 \%$ and $5.07 \%$, respectively) in comparison to that for OBs that were not treated with the inhibitor. Nevertheless, the inhibition of caveolae-mediated endocytosis and macropinocytosis did not result in the complete suppression of endocytosis. Caveolae-mediated endocytosis and micropinocytosis were highly involved in the uptake of S2.

\section{Intracellular localization of internalized S2}

Schmidt et $a .^{20}$ found that CaP nanoshells were internalized by cells and localized around the cell nucleus. Although research has confirmed that the nanoparticles may internalize, the subcellular distribution is still not clearly understood. To determine the intracellular distribution of internalized S2, the lysosomes and mitochondrion were labeled with a panel of specific organelle markers. In order to demonstrate the uptake of S2, OBs were cultured with S2 at $37^{\circ} \mathrm{C}$ for $24 \mathrm{~h}$ under cell culture conditions. CLSM was performed on the same cell areas (Fig. 4 and 5). The S2 nanoparticles were mainly incorporated
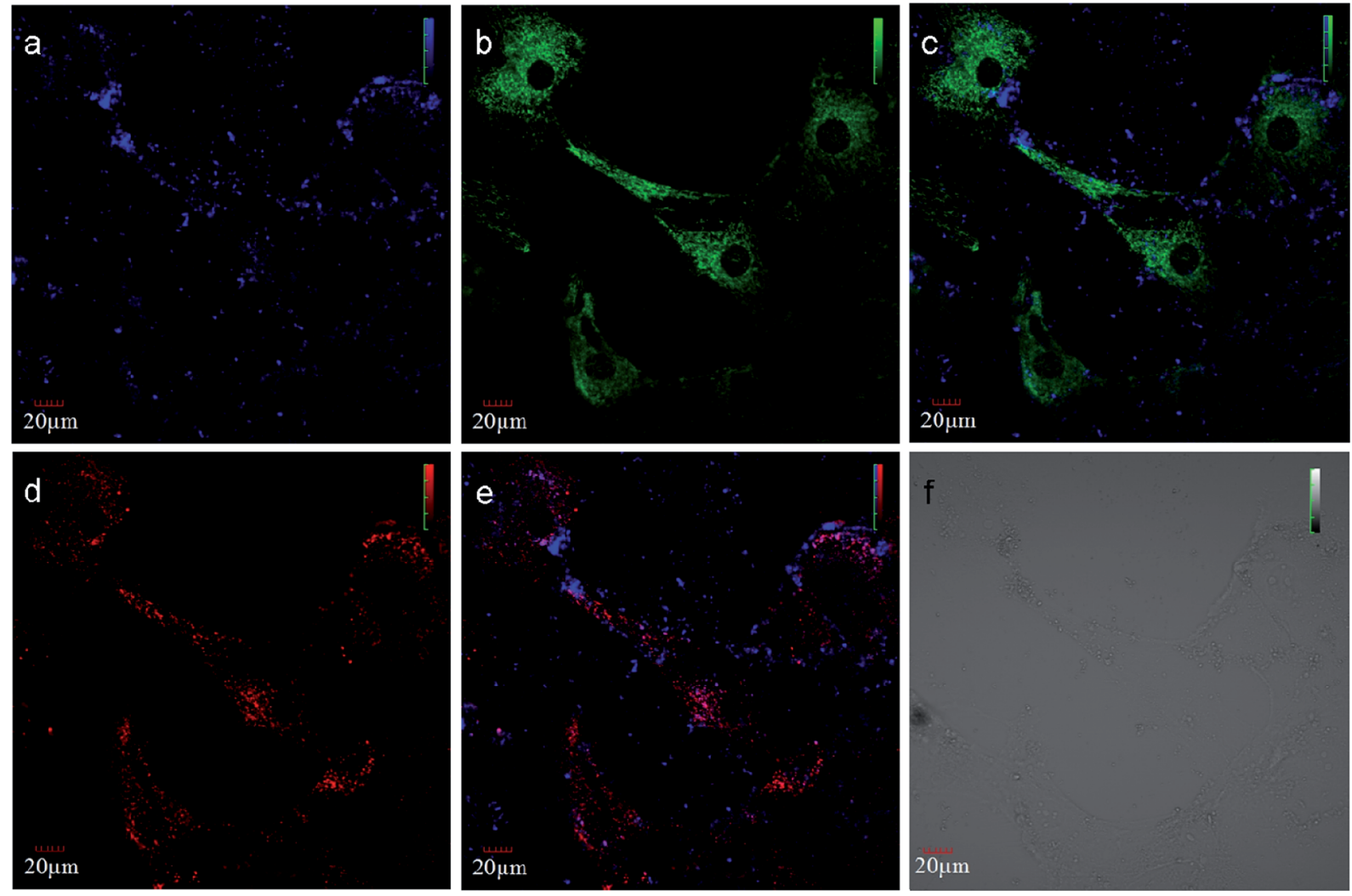

Fig. 4 Fluorescence images taken using CLSM. The cells were treated with S2 nanoparticles $\left(80 \mu \mathrm{g} \mathrm{mL}{ }^{-1}\right)$ for $24 \mathrm{~h}$. (a) Blue fluorescence $(425-$ $475 \mathrm{~nm}$ ) from S2 on excitation at $405 \mathrm{~nm}$ with a semiconductor laser beam; (b) green fluorescence (500-545 nm) from mitochondria with MitoTracker Green on excitation at $488 \mathrm{~nm}$ with an argon laser beam; (c) merged images of (a) and (c); (d) red fluorescence (575-675 nm) from lysosomes stained with Lyso-Tracker Red on excitation at $559 \mathrm{~nm}$ with a helium-neon laser beam; (e) merged images of (a) and (d); (f) bright field. Scale bar is $20 \mu \mathrm{m}$. 


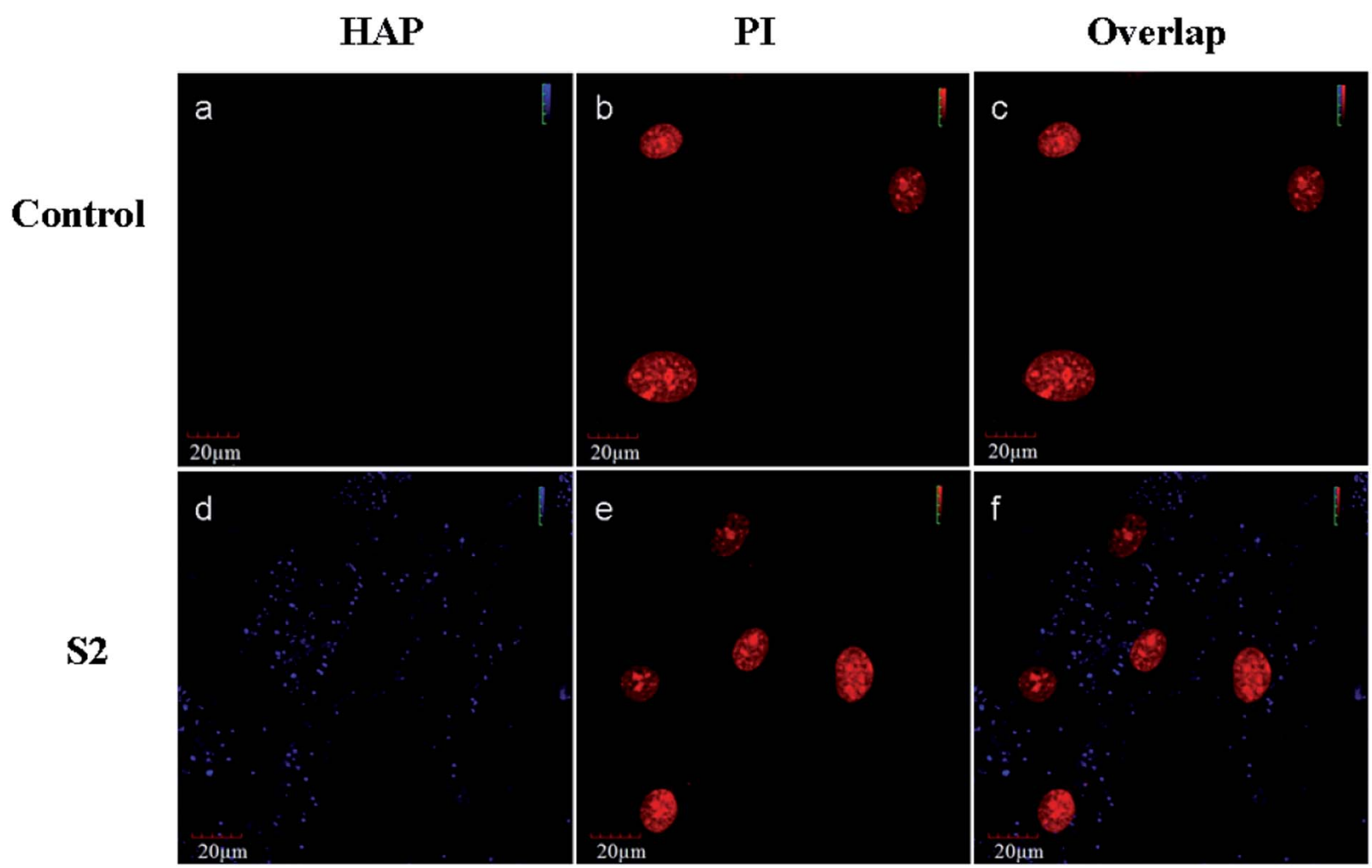

Fig. 5 Fluorescence images taken under CLSM. The cells were treated with S2 nanoparticles ( $\left.80 \mu \mathrm{g} \mathrm{mL}{ }^{-1}\right)$ for $24 \mathrm{~h}$. (a and d) Blue fluorescence $(425-475 \mathrm{~nm}$ ) from S2 on excitation at $405 \mathrm{~nm}$ with a semiconductor laser beam; (b and e) red fluorescence $(575-675 \mathrm{~nm})$ from nucleus stained with $\mathrm{Pl}$ on excitation at $559 \mathrm{~nm}$ with an argon laser beam; (c and f) overlap. Scale bar is $20 \mu \mathrm{m}$.

into the cytoplasm and not into the nucleus. As shown in Fig. 4 and 5, the S2 nanoparticles are mainly found to be associated with lysosomes areas (Fig. 4) but not inside the mitochondrion and the nucleus (Fig. 4 and 5). The HAP particles can be efficiently dissolved in the acidic intracellular compartments and release calcium ions. ${ }^{21} \mathrm{The}^{\mathrm{Ca}^{2+}}$ ions promote cell attachment, growth, and differentiation. ${ }^{22}$ Higher $\mathrm{Ca}^{2+}$ concentrations promote osteoblast differentiation, leading to bone mineralization in both growth and osteogenic conditions. ${ }^{23,24}$ The HAP particles internalized in the cells release $\mathrm{Ca}^{2+}$ and promote mineralization through an intracellular pathway.

\section{Effect of S2 on the formation of mineralized nodules}

A previous work has demonstrated that HAP enhances bone regeneration. ${ }^{25}$ On day 17 , S2 promoted the formation of the mineralized nodules of OBs at all concentrations in a dosedependent manner (Fig. 6).

\section{Effect of S2 on the synthesis of collagen of OBs}

Collagen I is widely used to evaluate osteogenic differentiation. ${ }^{26}$ As shown in Fig. 7, after incubating for $14 \mathrm{~d}$, S2 promotes the synthesis of collagen of OBs at the concentration
A

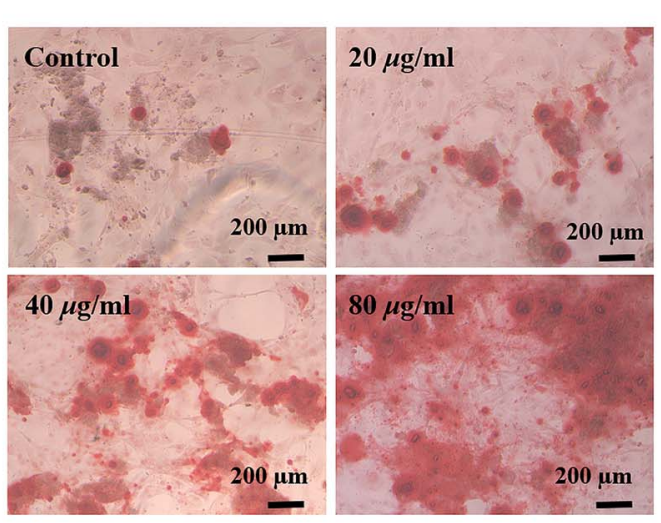

B

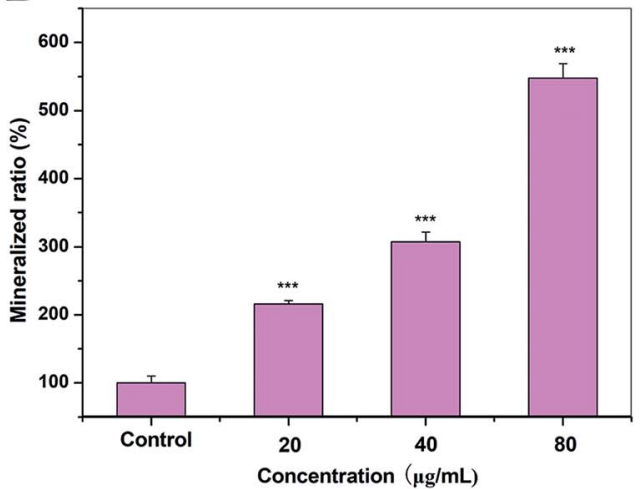

Fig. 6 Quantification of the mineralization in OBs cultured with S2. (A) Cells incubated with S2 nanoparticles were stained with alizarin red (ARS) after 17 days of culture. (B) The quantification (OD) of the destained ARS. Scale bar is $200 \mu \mathrm{m}$. 
A
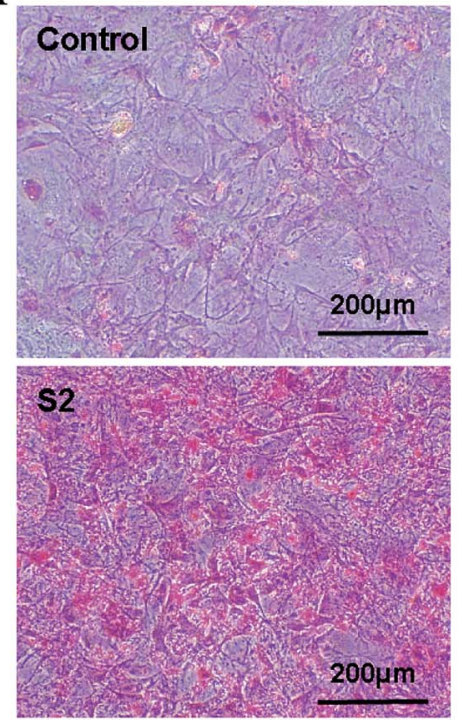

B

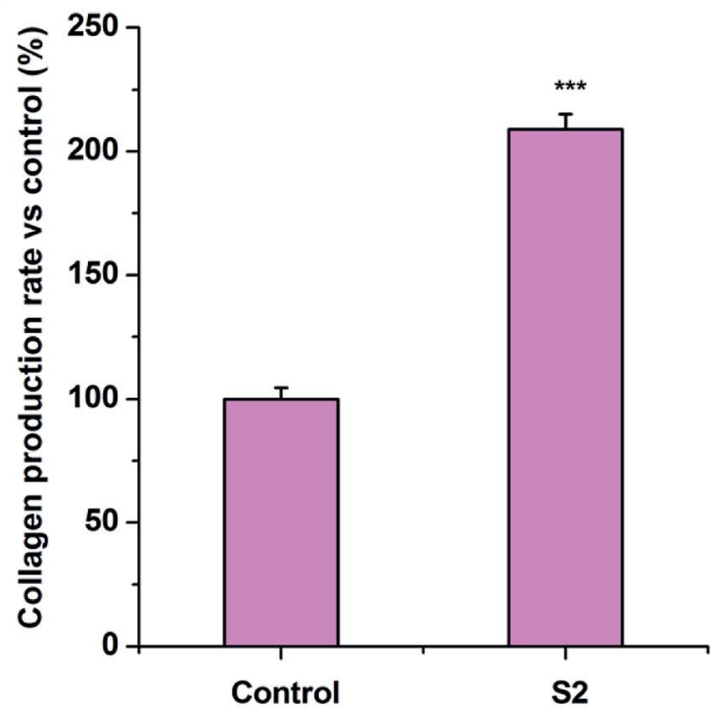

Fig. 7 Effect of S2 on the synthesis of collagen of OBs. OBs were treated with $\mathrm{S} 2\left(80 \mu \mathrm{g} \mathrm{mL}^{-1}\right)$ for $14 \mathrm{~d}$. The collagen was dyed by a Sirius red solution and quantified by a microplate spectrophotometer at a wavelength of $550 \mathrm{~nm}$. All results were normalized by the protein content. Data are presented as mean \pm SD from a representative of three separate experiments performed in sextuplicate. $* * * P<0.001$ compared with the control; the scale bar is $200 \mu \mathrm{m}$.

of $80 \mu \mathrm{g} \mathrm{mL} \mathrm{m}^{-1}$. The morphologic observation was in accordance with the results.

\section{Quantitative osteocalcin gene expression (RT-PCR analysis)}

The results for the OCN and RUNX2 gene expression over time are presented in Fig. 8. After 14 days of culture, the mRNA levels of RUNX2 and OCN were about 1.05- and 1.15-fold higher than that of control group, respectively. These results indicated that S2 up-regulated the expression of OCN, while it could not change the expression of RUNX2 under standard culture conditions. OCN is believed to be crucial in regulating osteoblast activity and binding of HAP. ${ }^{27}$ Combined with our Alizarin staining and Sirius red results, these results suggest that the

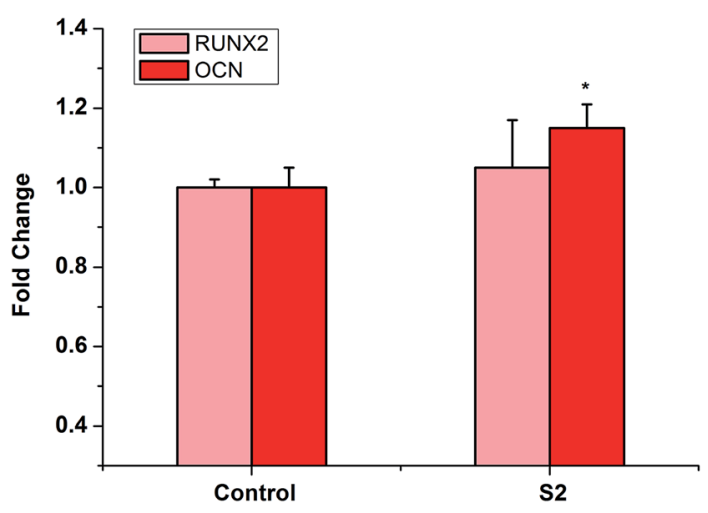

Fig. 8 Quantitative OCN and RUNX2 gene expressions after being cultured with S2. Data are presented as a fold ratio after being normalized to GAPDH values. $* P<0.05,{ }^{*} * P<0.01, * * * P<0.001$ vs. control, $n=3$. higher expression of OCN in the HAP nanoparticle-treated osteoblast cells stimulates more mineralization in the culture. ${ }^{28}$

\section{Nucleation role of $\mathrm{S} 2$ in the formation of the mineralized matrix}

Previous reports have demonstrated that a newly formed bone can be correlated with the presence of HAP nanoparticles, which can be associated with nucleating events. ${ }^{29}$ To date, there are no reports on whether the HAP particles promote bone regeneration intracellularly or extracellularly and whether they act as a mineral source or a nucleation site for mineral growth. ${ }^{20}$ The distribution of $\mathrm{S} 2$ in the mineralized nodule is shown in Fig. 9. The mineralized nodules produced by OBs have no luminescence, while $\mathrm{S} 2$ has defectrelated luminescence. By taking advantage of the fluorescence of S2, we found that extracellular S2 is located at the center of the mineralized nodules (Fig. 9). This result was consistent with previous results: the presence of HAP promoted the formation of mineralized nodules. The HAP particles can also promote mineralization through an extracellular pathway.

In summary, the S2 nanorods were uptaken by OBs through caveolae-mediated endocytosis and macropinocytosis and showed a positive effect in regulating the expression of OCN, the synthesis of collagen and the formation of mineralized nodules. Intracellular $\mathrm{S} 2$ could be distributed in lysosomes and dissolved and released calcium ions for the formation of mineralized nodules. The extracellular S2 as a nucleation site promoted the formation of mineralized nodules. The S2 nanorods promoted bone regeneration through both intracellular and extracellular pathways (Fig. 10). 


\section{UV excitation}
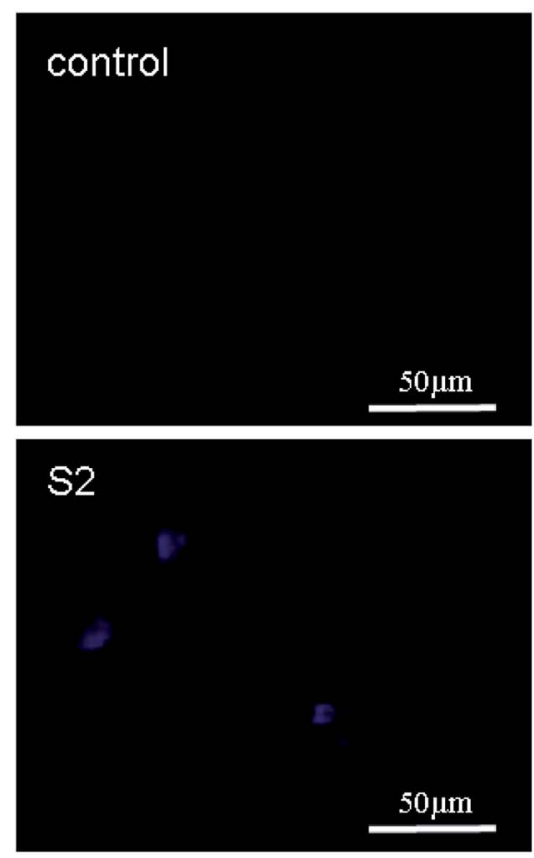

ARS staining
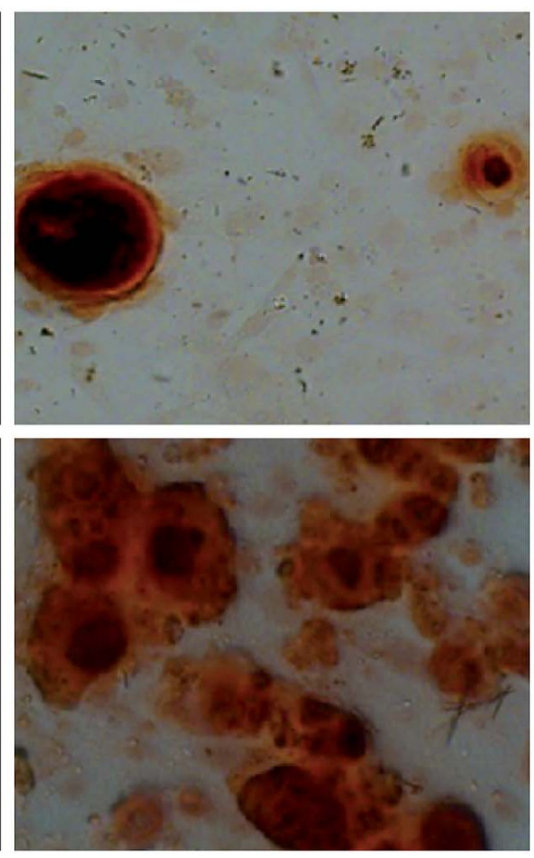

Merged
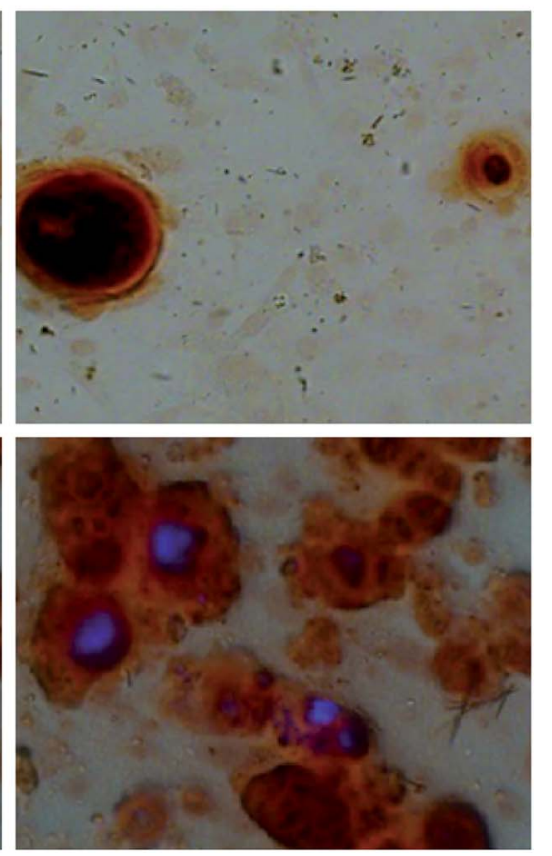

Fig. 9 Fluorescence imaging of mineralized nodules stained with alizarin red (ARS) after 17 days of culture. Blue, the blue light from S2 excited by UV; red, the bright image of the mineralized nodule; merged, the distribution of S2 in the mineralized nodule, the scale bar is $50 \mu \mathrm{m}$.

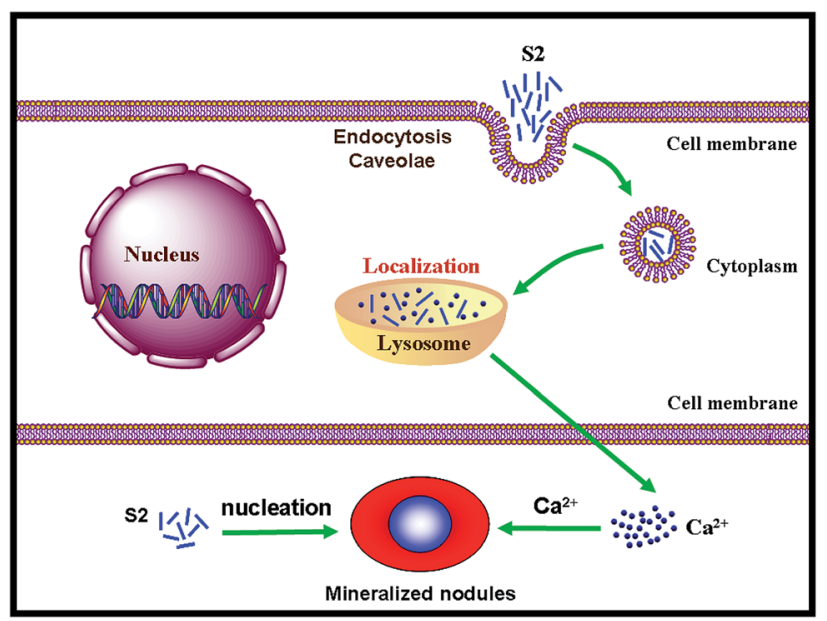

Fig. 10 The mechanism of S2 nanorods promoting OB mineralization through both intracellular and extracellular pathways.

\section{Conflicts of interest}

The authors declare no competing financial interest.

\section{Acknowledgements}

This research was supported by the Key Research and Development Plan of Hainan Province (Grant No. ZDYF2018160), National Natural Science Foundation of China (Grant No. 21766007), the Program for Innovative Research Team in
University (Grant No. IRT-16R19), Hainan Natural Science Foundation of China (Grant No. 217114), and the financial support from China Scholarship Council (CSC, Grant No. 201808460066, and 201808460067).

\section{References}

1 H.-W. Kim, J. C. Knowles, V. Salih and H.-E. Kim, J. Biomed. Mater. Res., Part B, 2004, 71, 66-76.

2 R. Pallela, J. Venkatesan, V. R. Janapala and S.-K. Kim, J. Biomed. Mater. Res., Part A, 2012, 100, 486-495.

3 S. Mirza, I. Zia, R. Jolly, S. Kazmi, M. Owais and M. Shakir, Int. J. Biol. Macromol., 2018, 119, 215-224.

4 A. Ashokan, D. Menon, S. Nair and M. Koyakutty, Biomaterials, 2010, 31, 2606-2616.

5 D. Li, Z. Liang, J. Chen, J. Yu and R. Xu, Dalton Trans., 2013, 42, 9877-9883.

6 Y. Li, X. Li, D. Wang, C. Shen and M. Yang, Microchim. Acta, 2018, 185, 435.

7 C. Zhang and J. Lin, Chem. Soc. Rev., 2012, 7938-7961.

8 S. Angelov, R. Stoyanova, R. Dafinova and K. Kabasanov, J. Phys. Chem. Solids, 1986, 47, 409-412.

9 W. H. Green, Science, 1997, 276, 1826-1828.

10 T. Brankova, V. Bekiari and P. Lianos, Chem. Mater., 2003, 15, 1855-1859.

11 W.-N. Wang, T. Ogi, Y. Kaihatsu, F. Iskandar and K. Okuyama, J. Mater. Chem., 2011, 21, 5183-5189.

12 C. Dai, J. Duan, L. Zhang, G. Jia, C. Zhang and J. Zhang, Biol. Trace Elem. Res., 2014, 162, 158-167. 
13 H.-S. Yu, J.-H. Jang, T.-I. Kim, H.-H. Lee and H.-W. Kim, J. Biomed. Mater. Res., Part A, 2009, 88, 747-754.

14 K. Amornwachirabodee, K. Chiablaem, S. Wacharasindhu, K. Lirdprapamongkol, J. Svasti, V. Vchirawongkwin and S. P. Wanichwecharungruang, J. Pharm. Sci., 2012, 101, 3779-3786.

15 C. Greulich, J. Diendorf, T. Simon, G. Eggeler, M. Epple and M. Köller, Acta Biomater., 2011, 7, 347-354.

16 M. N. M. Walter, A. Dehsorkhi, I. W. Hamley and C. J. Connon, Biomater. Sci., 2016, 4, 346-354.

17 T. Kaur and A. Thirugnanam, J. Mater. Sci. Technol., 2017, 33, 734-743.

18 I. W. Bauer, S.-P. Li, Y.-C. Han, L. Yuan and M.-Z. Yin, J. Mater. Sci.: Mater. Med., 2008, 19, 1091-1095.

19 Y. Cai, Y. Liu, W. Yan, Q. Hu, J. Tao, M. Zhang, Z. Shi and R. Tang, J. Mater. Chem., 2007, 17, 3780-3787.

20 S. M. Schmidt, K. A. Moran, A. M. T. Kent, J. L. Slosar, M. J. Webber, M. J. McCready, C. Deering, J. M. Veranth and A. Ostafin, J. Biomed. Mater. Res., Part A, 2008, 87, 418428.

21 R. D. Bloebaum, G. A. Lundeen, K. N. Bachus, I. Ison and A. A. Hofmann, J. Biomed. Mater. Res., 1998, 40, 104-114.
22 A. Bernhardt, A. Lode, C. Mietrach, U. Hempel, T. Hanke and M. Gelinsky, J. Biomed. Mater. Res., Part A, 2009, 90, 852-862. 23 S. Maeno, Y. Niki, H. Matsumoto, H. Morioka, T. Yatabe, A. Funayama, Y. Toyama, T. Taguchi and J. Tanaka, Biomaterials, 2005, 26, 4847-4855.

24 M. N. Lee, H. S. Hwang, S. H. Oh, A. Roshanzadeh, J. W. Kim, J. H. Song, E. S. Kim and J. T. Koh, Exp. Mol. Med., 2018, 50, 142.

25 S. Itoh, S. Nakamura, M. Nakamura, K. Shinomiya and K. Yamashita, Artif. Organs, 2006, 30, 863-869.

26 I. Bilic-Curcic, M. Kronenberg, X. Jiang, J. Bellizzi, M. Mina, I. Marijanovic, E. M. Gardiner and D. W. Rowe, Genesis, 2005, 43, 87-98.

27 S. Rammelt, M. Neumann, U. Hanisch, A. Reinstorf, W. Pompe, H. Zwipp and A. Biewener, J. Biomed. Mater. Res., Part A, 2005, 73, 284-294.

28 D. K. Khajuria, V. B. Kumar, D. Gigi, A. Gedanken and D. Karasik, ACS Appl. Mater. Interfaces, 2018, 10, 1937319385.

29 A. L. Rossi, I. C. Barreto, W. Q. Maciel, F. P. Rosa, M. H. Rocha-Leão, J. Werckmann, A. M. Rossi, R. Borojevic and M. Farina, Bone, 2012, 50, 301-310. 